\title{
Entwicklung medizinischer Fachabteilungen Identifikation von strategischen Erfolgsfaktoren zur langfristigen Ergebnissicherung
}

Welche strategischen Erfolgsfaktoren ein Reorganisationsprojekt einer medizinischen Fachabteilung zum langfristigen Erfolg führen, zeigen am anschaulichsten Best-Practice-Erfahrungen auf. Bei einem strategischen Erfolgsfaktor handelt es sich per Definition um eine Variable, „die den strategischen Erfolg langfristig maßgeblich zu beeinflussen vermag“ [1]. Wir wollen im Folgenden an dem Best-Practice-Beispiel einer kardiologischen Fachabteilung eines Universitätsklinikums diese Erfolgsfaktoren veranschaulichen.

\section{Erfolgsfaktor: Ganzheitliches}

Vorgehen - „Gemeinsam ein Zelt aufstellen"s

Medizinische Fachabteilungen im universitären Kontext können nach Mitarbeiteranzahl und Umsatz mit einem mittelständischen Unternehmen verglichen werden. Versteht sich diese Abteilung somit als Unternehmen im Unternehmen, ist logische Konsequenz, dass dort nicht einzelne Baustellen aufgerissen werden, ohne nach rechts und links zu schauen, sondern stets die Entwicklung der gesamten Abteilung als Unternehmung im Auge behalten wird.

Der Erfolg eines Reorganisationsprojektes begründet sich entscheidend aus seinem ganzheitlichen Ansatz. Um diesen von Beginn an zu verdeutlichen, hilft uns ein Bild: „Wir spannen gemeinsam ein Zelt“. Das unterstützt die eingebundenen Mitarbeiter darin, von Anfang an die Zusammenhänge zwischen den einzelnen Teilprojekten eines Reorganisationsprojektes zu verstehen. Unsere kardiologische
Fachabteilung besteht z. B. aus 4 zentralen Standbeinen. Übertragen auf die Metapher, sollen diese wie Zeltstangen gemeinsam im Rahmen eines Reorganisationsprojektes neu aufgestellt werden, damit das Zelt am Ende stabil steht. Konkret könnten solche Zeltstangen folgende Teilprojekte abbilden:

- Reorganisation der Stationsbetriebe und Standardisierung von Prozessabläufen

- Aufbau eines zentralen Bettenmanagements

- Reorganisation der Herzkatheterlabore in Verbindung mit den vor- und nachgelagerten Schnittstellen

- Reorganisation der Funktionsdiagnostik und Poliklinik

Welchen Kraftakt es für die Abteilung bedeutet, 4 solch zentrale Projekte gleichzeitig zu initiieren und umzusetzen, kann man sich vorstellen. Entscheidend ist es daher, den Mitarbeitern von Beginn an zu verdeutlichen, wie die einzelnen Projekte - die einzelnen Zeltstangen - ineinander
Tab. 1 Beispiel-Teilprojekte eines kardiologischen Reorganisationsprojektes.

1. Stationskonzept

- berufsgruppenübergreifender „Stundenplan“

- aufeinander abgestimmter Entlass- und Aufnahmeprozess

- gemeinsame Visiten

- Arztbriefschreibung

2. Konzept zum Belegungsmanagement

- Synchronisation von Herzkatheter- und Belegungsplanung

- definierte Bettenkontingente (elektiv, Notfall, interne Verlegungen)

3. Herzkatheter-Statut

- feste Zuordnung von Eingriffen zu den sechs Laboren

- High-Volume-Labor

- Staffelübergabe

- HK-Checkliste

4. Ambulanzkonzept

- zentrales Terminmanagement für die Funktionsbereiche

- Poliklinik-Organisation analog zur Arztpraxis

- Qualifizierung eines Ambulanzmanagers greifen und wie das Zelt am Ende der Umsetzung aussehen wird. Nur dadurch kann die notwendige Kraft und Motivation zur Umsetzung geschaffen werden.

\section{Erfolgsfaktor: Umsetzungsbeglei-} tung als zentraler Projektbaustein Das Projektvorgehen eines Reorganisationsprojektes besteht aus den 3 klassischen Projektphasen:

1. Ist-Analyse

2. Soll-Konzeption

3. Umsetzung

Unserer Erfahrung zufolge halten wir die Ist-Analyse bewusst kurz, da sich nicht lange mit der Vergangenheit aufgehalten, sondern der Blick auf das Hauptaugenmerk, die Veränderung, nach vorne gerichtet werden sollte. Eine Ist-Analyse ist dabei notwendig, um das Problembewusstsein zu schärfen und somit die Argumentation für die notwendige Veränderung zu legen. Methodisch setzt sie sich zusammen aus Prozessbeobachtungen, Interviews mit den Mitarbeitern und Datenauswertungen (u. a. Belegungsstatistiken). Gemeinsam mit dem Auftraggeber werden in dieser Phase Projektziel, Projektorganisation und Projektzeitplan definiert. Eine Projektleiter-Doppelspitze, bestehend aus einem internen Projektleiter (z.B. Oberarzt der Klinik) und einem externen Projektleiter, sorgt für eine fortlaufend enge Zusammenarbeit im Projekt. Für die einzelnen Teilprojekte werden berufsgruppenübergreifende Projektgruppen benannt; bei Schnittstellenthemen werden zudem Vertreter aus den angrenzenden Bereichen eingeladen. Eine Kick-Off-Veranstaltung mit allen Leitungskräften der Fachabteilung (Oberärzte und Stationsleitungen) rundet die Ist-Analyse ab.

In berufsgruppenübergreifenden Projektgruppensitzungen findet die parallele Soll-Konzepterstellung statt. So lassen sich für die 4 Teilprojekte z.B. Schwerpunktthemen nennen (Tab. 1).

Entscheidend bei der Soll-Konzeptionierung ist die berufsgruppenübergreifende Zusammenarbeit, die sicherstellt, dass 
die Konzepte in jeder Berufsgruppe Akzeptanz und später Anwendung finden. Zu Beginn ist die Zusammenarbeit in den Projektgruppen oftmals von Schwierigkeiten geprägt, die es gilt, Schritt für Schritt aufzulösen. So muss z. B. eine gemeinsame Sprache gefunden, die unterschiedlichen Erwartungen abgeglichen und auf einen gemeinsamen Nenner gebracht und ein stückweit die Zusammenarbeit wieder neu erlernt werden. Lassen sich diese Grundsteine in der Projektgruppenarbeit legen, wirken sie lange positiv für den Projekterfolg nach. Unsere Erfahrung zeigt, dass die berufsgruppenübergreifende Kommunikation auch nach Projektende bestehen bleibt und darauf basierend aufkommende Probleme über kurze Abstimmungswege gemeinsam gelöst werden.

Nach Freigabe der Konzepte durch den Klinikdirektor und die Pflegedienstleitung findet eine umfassende Information der Mitarbeiter der Fachabteilung statt. Wichtig ist, dass zeitnah mit der Umsetzung begonnen wird, damit die Mitarbeiter sehen, dass es voran geht, dass nicht nur geredet, sondern auch gehandelt wird. Eine enge externe Begleitung in den ersten 3 Monaten trägt unseren Erfahrungen nach viel zum Umsetzungserfolg bei. Anhand von Prozesskennzahlen, die zu Beginn täglich erfasst werden (z. B. Feedback-Bögen auf Station, auf denen alle Aufnahmen (Bett frei?), Entlassungen (Einhaltung von Zeitzielen) und Visiten (Pünktlicher Beginn? Teilnahme der Pflege?) dokumentiert werden), stehen neben Prozessbegleitungen, Begehungen und Interviews weitere Evaluationsinstrumente zur Verfügung. Erfolgreiche Prozessoptimierungsprojekte zeichnen sich dadurch aus, dass die Phase nach Konzepterstellung mindestens genauso wichtig, wenn nicht noch wichtiger angesehen wird als die Phase davor. Entscheidend in dieser Phase sind frühe Erfolge, um die Motivation zu steigern und kritischen Stimmen begegnen zu können.

\section{Erfolgsfaktor: Strategische und operative Verant-} wortungsübernahme durch das Leitungsteam

Nicht nur die Prozesse der Fachabteilung sollten bei einem Reorganisationsprojekt im Fokus stehen, sondern auch die strategische Ausrichtung der Klinik. Unter Einbindung seiner Oberärzte sollte ein Chefarzt die Vision und Werte der Abteilung festschreiben und die medizinischen Schwerpunkte definieren. Zudem empfiehlt sich, die oberärztlichen Verantwortungsbereiche mithilfe von Organigrammen klar festzulegen, welche die Strukturen von Personal-, Produkt- und Ressortverantwortung abbilden.

Die Rahmenbedingungen für die Personalverantwortung steckt ein Personalentwicklungskonzept für den ärztlichen Dienst, welches u. a. ein Mentorensystem und das Curriculum zur Rotationsplanung enthält. Das Organigramm bildet das Mentorensystem ab, indem es die Assistenzärzte den Oberärzten zuordnet. Mitarbeiterführung auf Oberarztebene ist zweifach entscheidend, zum einen um in der persönlichen Karriere auf die Führungsrolle vorzubereiten, zum anderen unter Aspekten der Mitarbeitergewinnung und -bindung. Assistenzärzten, denen eine klare Perspektive und Führung geboten wird, entscheiden sich in den aktuellen Zeiten des Ärztemangels leichter für eine Abteilung. Für die Rotationsplanung gilt es daher, ein festes Schema zu entwickeln, das sicherstellt, dass die Assistenzärzte eine gemäß 
Ärztekammer umfängliche und zeitgerechte Ausbildung erhalten, und das den Ansprüchen der Klinik gerecht wird. Das Curriculum-Schema und festgelegte Planungszeitpunkte erlauben eine langfristige Organisation für Assistenzärzte und auch die Klinik.

Die Produktverantwortung bezieht sich auf das medizinische Leistungsportfolio. Dieses wird unterteilt in einzelne Schwerpunkte bzw. Produkte, für die jeweils Oberärzte die Verantwortung übernehmen. Die produktverantwortlichen Oberärzte sollen für ihren Schwerpunkt jeweils eine Balanced Scorecard (BSC) erstellen, welche die strategischen Stoßrichtungen, kritischen Erfolgsfaktoren und strategischen Maßnahmen enthält. Die BSCs sollten dann im Leitungsteam (Chefarzt, Oberärzte) abgestimmt werden, sodass auch hier ein ganzheitliches medizinisches Leistungsportfolio entstehen kann.

Die Ressortverantwortung bezieht sich auf organisatorische Bereiche, wie z. B. Station, Ambulanz, IT, Personal, Marketing. Aufgabe der Ressortverantwortlichen ist es, einen jährlichen Ressortplan zu erstellen, dessen Umsetzung am Jahresende analog $\mathrm{zu}$ Zielvereinbarungen gemeinsam mit dem Chefarzt evaluiert wird.

Alle 3 Verantwortungsstrukturen sind in regelmäßigen Abständen feste Tagesordnungspunkte im Leitungsteam; auch eine jährliche Strategieklausur dient der gemeinsamen strategischen Abteilungsentwicklung. Die enge Einbindung der Oberärzte fördert die Akzeptanz und gleichzeitig stellt sie die Verantwortungsübernahme sicher, was zwingende Voraussetzung für den nächsten Erfolgsfaktor ist.

\section{Erfolgsfaktor: Etablierung selb-} ständiger berufsgruppenübergreifender Feedback-Strukturen

Im Zuge der Festigung von neu definierten Prozessen gilt es, Strukturen für eine langfristige und eigenständige Sicherung zu schaffen. „Wollen - Können - Lassen“ beschreibt einen erfolgreich erprobten Dreisatz zum Aufbau von Kompetenz und Selbstvertrauen. Berufsgruppenübergreifende Führungskräfte-Coachings beschäftigen sich mit den Themen Motivation, Überwindung von Widerständen und Selbstmanagement und setzen den Grundstein zum „Wollen“. Wollen alleine reicht jedoch nicht aus, um langfristig neue Prozesse aufrecht zu halten. Das nötige Methodenwissen muss von uns an den Kunden transferiert werden, damit er „kann“. Dies geschieht in den Projekten u. a. durch Informationsveranstaltungen für alle Mitarbeiter, im Projektfortschritt durch gemeinsame Moderation von Projektgruppen und damit dem Erlernen von Moderationstechniken und durch die Zurverfügungstellung von Vorlagen, z. B. für Protokolle, die eine Maßnahmenumsetzung erleichtern, indem sie Verantwortlichkeiten, Zeitziele, Wiedervorlage etc. klar definieren. Im dritten Schritt geht es noch um die Wissensanwendung; entscheidend ist, dies zu zu-,,lassen“. Ein langsamer Rückzug von einer zunächst engen externen Kontrolle zu Beginn hin zu einem regelmäßigen Feedback gegen Ende ermöglicht dabei einen zunehmenden Transfer der Verantwortung.

Der Ansatz, eine selbständige berufsgruppenübergreifende Feedback-Struktur zu etablieren, erleichtert diesen Transfer: Die Projektgruppen der einzelnen Teilprojekte (aus dem Beispiel oben: Station, Bettenmanagement, Herzkatheter, Ambulanz) tagen z. B. einmal pro Monat, um $\mathrm{zu}$ evaluieren, was gut läuft und was nicht. Bei Verbesserungspotenzialen werden umgehend Maßnahmen ergriffen und im Protokoll festgehalten, welches beim nächsten Mal auf Wiedervorlage kommt. Zudem gibt es eine Schnittstellen-Feedbackrunde, die aus Vertretern der anderen Gruppen besteht, sodass auch Schnittstellenprobleme unmittelbar eigenständig und bereichs- sowie berufsgruppenübergreifend gelöst werden. Übergeordnet verantwortlich für das Feedback-Management ist der interne Projektleiter, der gemeinsam mit einer Stationsleitung eine berufsgruppenübergreifende Doppelspitze bilden sollte.

\section{Erfolgsfaktor: Kontinuierliche Überwachung und Weiterent- wicklung}

Nach Projektabschluss dienen (z. B. halbjährliche) externe Prozessaudits, sog. Feedback-Wochen, bestehend aus Feedback-Runden, Begehungen und Auswertungen von Prozesskennzahlen, der kontinuierlichen Überwachung und Weiterentwicklung der Abteilung. Abschluss einer Feedback-Woche bildet die Vorstellung der Ergebnisse im Gesamtleitungsteam (Chefarzt, Pflegedienstleitung, Oberärzte, leitende Stationsärzte, Stationsleitungen) begleitet von einem detail- lierten Ergebnisbericht und Maßnahmenkatalog.

Ein erfolgreiches Reorganisationsprojekt unterscheidet sich vor allem in 2 Aspekten deutlich von anderen Projekten dieser Art: Der ganzheitliche Ansatz verbunden mit einer von Beginn an berufsgruppenübergreifenden Zusammenarbeit. Kommt als zweiter Erfolgsfaktor die erfolgreiche Verantwortungsübernahme der Leitungskräfte hinzu, steht einer langfristigen Ergebnissicherung nichts mehr im Wege.

\section{Literatur}

1 Grünig R, Heckner F, Zeuss A. Methoden zur Identifikation strategischer Erfolgsfaktoren. Die Unternehmung 1996, (50) (1): S 3-12.

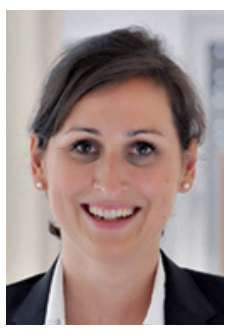

Korrespondenz Dr. Mirjam Pföhler Ruhl Consulting AG Harrlachweg 1 68163 Mannheim E-Mail: mirjam.pfoehler@ ruhl-consulting.dew 